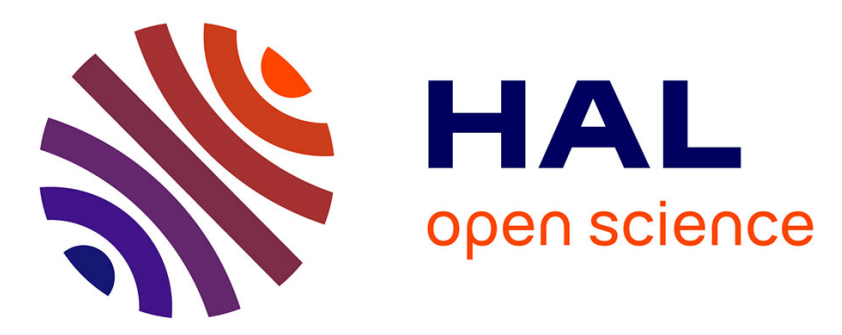

\title{
Mesure de l'atténuation et de la vitesse de propagation ultrasonore par une technique spectroscopique
}

\author{
J.R. Emery, M. Tabellout
}

\section{To cite this version:}

J.R. Emery, M. Tabellout. Mesure de l'atténuation et de la vitesse de propagation ultrasonore par une technique spectroscopique. Revue de Physique Appliquée, 1990, 25 (2), pp.243-251. 10.1051/rphysap:01990002502024300 . jpa-00246182

\section{HAL Id: jpa-00246182 https://hal.science/jpa-00246182}

Submitted on 1 Jan 1990

HAL is a multi-disciplinary open access archive for the deposit and dissemination of scientific research documents, whether they are published or not. The documents may come from teaching and research institutions in France or abroad, or from public or private research centers.
L'archive ouverte pluridisciplinaire HAL, est destinée au dépôt et à la diffusion de documents scientifiques de niveau recherche, publiés ou non, émanant des établissements d'enseignement et de recherche français ou étrangers, des laboratoires publics ou privés. 


\title{
Mesure de l'atténuation et de la vitesse de propagation ultrasonore par une technique spectroscopique
}

\author{
J. R. Emery et M. Tabellout \\ Laboratoire de Physique de l'Etat Condensé - Ultrasons, U.R.A. n $^{\circ} 807$, Faculté des Sciences, Université du \\ Maine, Route de Laval, 72017 Le Mans Cedex, France
}

(Reçu le 28 juillet 1989, révisé et accepté le 9 novembre 1989)

\begin{abstract}
Résumé. - Une spectroscopie ultrasonore par F.F.T. est présentée qui permet de mesurer la vitesse et l'absorption des ondes ultrasonores dans les milieux condensés sur une large gamme de fréquences. La validation expérimentale de cette technique est testée sur des liquides connus par comparaison avec les grandeurs obtenues par les méthodes ultrasonores classiques, comme le 1.2.2 trichloroéthane ou certains mélanges aqueux d'alcools. Cette technique est utilisée également pour la détermination de l'évolution dans le temps des spectres de relaxation des systèmes qui évoluent, en particulier pour ceux dont la croissance est gouvernée par des processus d'agrégation.

Abstract. - A technique of F.F.T. ultrasonic spectroscopy is described which allows determination of the absorption and velocity of sound in the condensed phase over a wide frequency range. The experiment was checked using a comparison of these data and those obtained using classical ultrasonic methods on 1.1.2 trichloroethane and certain aqueous mixtures of alcohol. The use of this technique to study the change in relaxation spectrum for systems changing with time and in particular those undergoing gelation are described.
\end{abstract}

\section{Introduction.}

La spectroscopie ultrasonore est l'étude de la propagation des ultrasons dans le domaine de fréquence de $20 \mathrm{kHz}$ à $1000 \mathrm{MHz}$. Les caractéristiques de ces ondes (longitudinales et transversales) sont une composante importante de la connaissance des propriétés mécaniques et structurales du milieu dans lequel elles se propagent, tant à l'échelle macroscopique qu'à l'échelle moléculaire.

A l'échelle macroscopique, les ultrasons peuvent être sensibles aux défauts ou aux discontinuités de la matière (contrôle non destructif), au grain du matériau ou à la qualité d'adhérence d'un collage.

A l'échelle moléculaire, l'évolution des grandeurs caractéristiques (absorption, vitesse, modules élastiques) en fonction de la fréquence montre la réponse du milieu à une excitation mécanique ; cette réponse dépend des interactions inter et intra-moléculaires et peut être interprétée en termes de structure.

Les grandeurs sont mesurées à des fréquences fixes et une exploration en fréquence peut prendre un temps assez long. La spectroscopie par Transfor- mée de Fourier Rapide permet une exploration en fréquence très rapide (dans la limite de la bande passante du traducteur) au moyen d'une impulsion ultrasonore au spectre aussi large que possible et qui interagit avec le milieu d'étude.

Les progrès récents effectués dans le domaine de l'acquisition et du traitement du signal permettent d'atteindre des fréquences de plusieurs centaines de $\mathrm{MHz}$.

Le propos de la présente étude est de présenter une technique de spectroscopie ultrasonore à Transformée de Fourier Rapide dans une gamme de fréquence de 1 à $400 \mathrm{MHz}$ (étendue à $1000 \mathrm{MHz}$ ) et son application à la mesure de l'absorption et de la vitesse de propagation des ultrasons dans les milieux condensés, liquides, solides et gels. Le bon accord entre les mesures effectuées par cette méthode et celles effectuées par interférométrie classique montre la fiabilité de la méthode et autorise la détermination de processus de relaxation dans les solides (amorphes ou cristallins), processus difficilement descriptibles par d'autres méthodes d'investigation. 
1. Propagation des ondes ultrasonores dans les milieux condensés isotropes.

Les ondes élastiques qui se propagent dans un milieu condensé isotrope de masse volumique $\rho$ sont soit transversales (déplacement $Y$ des particules perpendiculaire à la direction $x$ de propagation) soit longitudinales (déplacement $X$ parallèle à la direction $x$ de propagation). Les équations de propagation de chacun des systèmes d'ondes sont respectivement :

$$
\frac{\partial^{2} Y}{\partial t^{2}}=\frac{G^{*}}{\rho} \cdot \frac{\partial^{2} Y}{\partial x^{2}}
$$

et

$$
\frac{\partial^{2} X}{\partial t^{2}}=\frac{M^{*}}{\rho} \cdot \frac{\partial^{2} X}{\partial x^{2}}
$$

$G^{*}$ et $M^{*}$ sont respectivement les modules complexes de cisaillement et longitudinal.

Dans l'hypothèse de l'acoustique linéaire et d'une excitation sinusoïdale de pulsation $\omega$, la solution de l'équation de propagation des ondes transversales (1) est de la forme :

$$
Y=Y_{0} \exp \left(-\alpha_{\mathrm{T}} x\right) \exp \left(-j \omega\left(t-\frac{x}{v_{\mathrm{T}}}\right)\right) .
$$

En général, la vitesse de propagation et l'absorption de l'onde transversale dépendent de la fréquence :

$$
v_{\mathrm{T}}^{2}=\frac{\eta_{\mathrm{s}}}{\rho} \frac{\omega^{2} \tau_{\mathrm{s}}}{1+\omega^{2} \tau_{\mathrm{s}}^{2}} \quad \frac{\alpha_{\mathrm{T}}}{\omega^{2}}=\frac{1}{2 \rho v_{\mathrm{T}}^{3}} \frac{\eta_{\mathrm{s}}}{1+\omega^{2} \tau_{\mathrm{s}}^{2}}
$$

en supposant $\left(\alpha_{\mathrm{T}} v_{\mathrm{T}} / \omega\right)^{2} \ll 1$ et en définissant le coefficient de viscosité de cisaillement $\eta_{\mathrm{s}}$ comme suit :

$$
\eta_{\mathrm{s}}=\tau_{\mathrm{s}} G
$$

$G$ et $\tau_{\mathrm{s}}$ sont respectivement le module et le temps de relaxation de cisaillement.

L'équation de propagation des ondes longitudinales, solution de l'équation (2), est dans les mêmes conditions d'approximation, de la forme :

$$
X=X_{0} \exp \left(-\alpha_{\mathrm{L}} x\right) \exp \left(-j \omega\left(t-\frac{x}{v_{\mathrm{L}}}\right)\right) .
$$

La vitesse et l'absorption des ondes de compression dépendent également de la fréquence et si $K_{0}$ est la limite à fréquence nulle du module de compression on a :

$$
\begin{gathered}
v_{\mathrm{L}}^{2}=\frac{1}{\rho}\left(K_{0}+\eta_{\mathrm{v}} \frac{\omega^{2} \tau_{\mathrm{v}}}{1+\omega^{2} \tau_{\mathrm{v}}^{2}}+\frac{4}{3} \eta_{\mathrm{s}} \frac{\omega^{2} \tau_{\mathrm{s}}}{1+\omega^{2} \tau_{\mathrm{s}}^{2}}\right) \\
\frac{\alpha_{\mathrm{L}}}{\omega^{2}}=\frac{1}{2 \rho v_{\mathrm{L}}^{3}}\left(\frac{\eta_{\mathrm{v}}}{1+\omega^{2} \tau_{\mathrm{v}}^{2}}+\frac{4}{3} \frac{\eta_{\mathrm{s}}}{1+\omega^{2} \tau_{\mathrm{s}}^{2}}\right)
\end{gathered}
$$

expressions dans lesquelles $\eta_{\mathrm{v}}$ est le coefficient de viscosité de volume défini d'une manière semblable à $\eta_{\mathrm{s}}$ mais à partir des limites à fréquence nulle $K_{0}$ et à fréquence infinie $K_{\infty}$ du module de compressibilité de volume $K$ :

$$
\eta_{\mathrm{v}}=\left(K_{\infty}-K_{0}\right) \tau_{\mathrm{v}}
$$

$\tau_{\mathrm{v}}$ est le temps de relaxation de volume.

L'absorption ultrasonore des ondes longitudinales comporte donc un terme de viscosité de volume et un terme de viscosité de cisaillement.

Les phénomènes de relaxation sont dus à l'énergie de translation acquise par les molécules du milieu lors des augmentations de température causées par les augmentations de pression de l'onde; cette énergie est échangée au moyen d'un mécanisme de couplage inter et intramoléculaire sous d'autres formes, comme un changement de configuration ou de structure par exemple [2]. Ces comportements sont facilement expliqués lorsqu'ils relèvent d'un modèle simple comme dans le cas des différents niveaux d'énergie des isoméries rotationnelles dans le 1.1.2 trichloroéthane [3].

Toutefois dans la plupart des milieux viscoélastiques, plusieurs processus sont possibles et il est nécessaire de prendre en compte plusieurs temps de relaxation, voire une distribution $f(\tau)$ de ces temps ; la partie relaxante s'exprime alors sous la forme:

$$
\int_{-\infty}^{+\infty} f(\tau) \mathrm{d} \tau /\left(1+\omega^{2} \tau^{2}\right) .
$$

Dans le cas de fluides très visqueux, les domaines de relaxation peuvent se superposer comme dans le cas de l'éthanol à basse température [4].

Les valeurs d'absorption et de vitesse mesurées pour des fréquences discrètes sont habituellement ajustées à des courbes de relaxation correspondant à des modèles qui expriment des processus à plusieurs temps, ou à une distribution de temps de relaxation. Le balayage en fréquence nécessite alors un temps d'autant plus grand que le nombre de fréquences expérimentales est élevé. D'autre part, si le milieu étudié est susceptible d'évoluer dans le temps comme par exemple dans le cas d'un milieu soumis à une réaction chimique ou à une transition de phase [5], l'évolution de l'absorption et de la vitesse de propagation ne peut être suivie qu'à une seule fréquence. L'évolution des paramètres d'un processus de relaxation nécessite la connaissance de l'évolution de ces grandeurs dans un domaine fréquentiel aussi grand que possible, d'où l'intérêt de l'utilisation d'une méthode de spectroscopie ultrasonore par F.F.T. [6, 7].

\section{Principe de la méthode.}

La solution des équations de propagation d'une onde polychromatique s'exprime par intégration, sur 
tout le domaine de fréquence, de l'équation (3) pour une onde de cisaillement ou de l'équation (4) pour une onde de pression. En considérant la propagation d'une onde de pression, l'amplitude de déplacement des particules s'écrit :

$$
\begin{aligned}
X(x, t)=\int_{-\infty}^{+\infty} A_{0}\left(\nu^{\prime}\right) \exp (-\alpha x) \times \\
\quad \times \exp \left(-j 2 \pi \nu^{\prime}\left(t-\frac{x}{v}\right)\right) \mathrm{d} \nu^{\prime} .
\end{aligned}
$$

La forme mathématique est semblable pour une onde de cisaillement.

La transformée de Fourier de $X(x, t)$ est :

$$
\begin{gathered}
\mathcal{F}[X(x, t)]=\int_{-\infty}^{+\infty} \int_{-\infty}^{+\infty} A_{0}\left(\nu^{\prime}\right) \exp (-\alpha x) \times \\
\times \exp \left(-j 2 \pi \nu^{\prime}\left(t-\frac{x}{v}\right)\right) \mathrm{d} \nu^{\prime} \exp (j 2 \pi \nu t) \mathrm{d} t \\
=A_{0}(\nu) \exp (-\alpha(\nu) x) \exp \left(j \frac{2 \pi \nu}{v} x\right)
\end{gathered}
$$

L'atténuation ultrasonore est caractérisée par l'amplitude :

$$
A(\nu)=A_{0}(\nu) \exp (-\alpha(\nu) x)
$$

et la vitesse par la phase :

$$
\varphi(\nu)=\frac{2 \pi \nu}{v(\nu)} x
$$

Le principe de la méthode consiste à calculer la vitesse de phase et l'amortissement du milieu étudié à partir des arguments et des modules des transformées de Fourier des signaux transmis [8] dans la bande des fréquences autorisée par les traducteurs piézo-électriques utilisés.

Les composants d'un système de mesures ultrasonores sont constitués d'un générateur qui excite le traducteur piézoélectrique émetteur lequel transforme le signal en onde ultrasonore qui se propage dans une ligne à retard; le traducteur récepteur reconvertit ce signal après transit dans le milieu d'étude en signal électrique qui est amplifié et traité. La figure 1 présente les éléments d'un système spectroscopique ultrasonore.

Le signal de sortie $v_{2}(t)$ est lié au signal d'entrée $v_{1}(t)$ par la relation :

$$
v_{2}(t)=v_{1}(t) * h_{1}(t) * h(t) * h_{2}(t)
$$

ou $*$ est le symbole du produit de convolution et :

$$
h_{1}(t)=b_{1}(t) * x_{1}(t) * c_{1}(t)
$$

et

$$
h_{2}(t)=c_{2}(t) * x_{2}(t) * b_{2}(t) * a(t) * g(t) * d(t) .
$$

De même dans le domaine fréquentiel on a :

$$
V_{2}(\nu)=V_{1}(\nu) \cdot H_{1}(\nu) \cdot H(\nu) \cdot H_{2}(\nu) \text {. }
$$

Réponse temporelle

Réponse fréquentielle

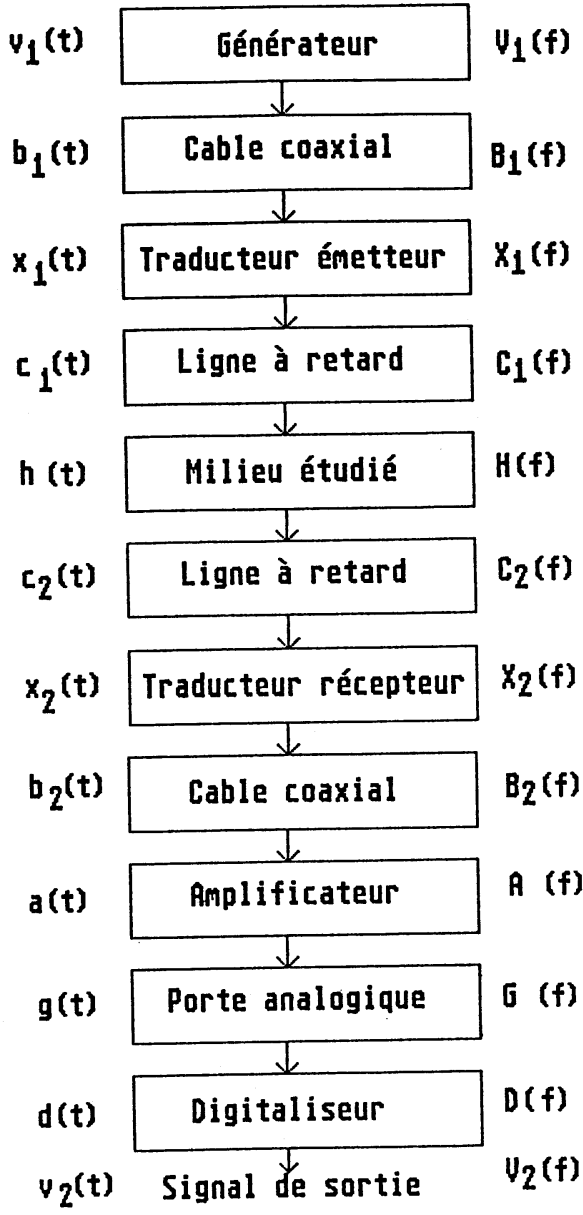

Fig. 1. - Eléments d'un système spectroscopique ultrasonore.

[Elements of an ultrasonic spectroscopy system.]

Le principe de mesure consiste à extraire la fonction de transfert $H(\nu)$ à partir de la transformée de Fourier du signal de sortie $V_{2}(\nu)$ par deux méthodes différentes suivant qu'il s'agit de liquides stables ou de systèmes qui, soumis à une réaction chimique, voient leurs propriétés mécaniques évoluer au cours du temps.

Dans le premier cas la fonction de transfert s'obtient en considérant la transformée de Fourier du signal de sortie pour deux distances différentes $x_{1}$ et $x_{2}$ :

$$
\alpha(\nu)=\frac{1}{\left(x_{2}-x_{1}\right)} \operatorname{Ln}\left(\left|\frac{V_{2}\left(\nu, x_{2}\right)}{V_{2}\left(\nu, x_{1}\right)}\right|\right)
$$

et

$$
v(\nu)=\frac{2 \pi \nu\left(x_{2}-x_{1}\right)}{\Phi_{2}(\nu)-\Phi_{1}(\nu)} .
$$

La différence de phase $\Delta \Phi=\Phi_{2}-\Phi_{1}$ tient compte du temps de transit $\Delta t$ induit par la variation 
de distance et de la dispersion de phase $\Delta \varphi$ qui en résulte.

Dans le cas de systèmes qui évoluent vers une structure solide il est alors nécessaire d'utiliser un liquide de référence dont on connaît la fonction de transfert $H_{\mathrm{r}}(\nu)$.

Soit $H_{t}(\nu)$ la fonction de transfert du système étudié à l'instant $t$. La première mesure effectuée sur le liquide de référence donne un signal de sortie dont la transformée de Fourier est :

$$
V_{2 \mathrm{r}}(\nu)=H_{1 \mathrm{r}}(\nu) \cdot H_{\mathrm{r}}(\nu) \cdot H_{2 \mathrm{r}}(\nu) .
$$

A l'instant $t$ la mesure effectuée sur le milieu étudié donne un signal de sortie dont la transformée de Fourier est :

$$
V_{2 t}(\nu)=H_{1 t}(\nu) \cdot H_{t}(\nu) \cdot H_{2 t}(\nu) .
$$

A partir de ces deux mesures on obtient la fonction de transfert du milieu étudié à l'instant $t$ :

$$
H_{t}(\nu)=\frac{V_{2 t}(\nu)}{V_{2 \mathrm{r}}(\nu)} H_{\mathrm{r}}(\nu) \cdot \beta
$$

avec

$$
\beta=\frac{H_{1 t}(\nu) \cdot H_{2 t}(\nu)}{H_{1 \mathrm{r}}(\nu) \cdot H_{2 \mathrm{r}}(\nu)}=\frac{Z_{t}\left(Z_{1}+Z_{\mathrm{r}}\right)^{2}}{Z_{\mathrm{r}}\left(Z_{1}+Z_{t}\right)^{2}}
$$

où $Z_{1}, Z_{\mathrm{r}}$ et $Z_{t}$ sont les impédances acoustiques respectives des lignes à retard du liquide de référence et du milieu étudié à l'instant $t$. De ces deux mesures on peut déduire l'absorption et la vitesse des ondes ultrasonores :

$$
\alpha(\nu)=\alpha_{\mathrm{r}}(\nu)+\frac{1}{x} \operatorname{Ln}\left(\left|\frac{V_{2 t}(\nu)}{V_{2 \mathrm{r}}(\nu)}\right| \beta\right)
$$

et

$$
v(\nu)=\frac{\omega \cdot x \cdot v_{\mathrm{r}}}{\omega x+v_{\mathrm{r}}\left(\Phi_{t}-\Phi_{\mathrm{r}}\right)}
$$

$\alpha_{\mathrm{r}}(\nu), \Phi_{\mathrm{r}}(\nu)$ et $v_{\mathrm{r}}$ sont relatifs au liquide de référence.

\section{Dispositif expérimental.}

3.1 EMETTEUR, RÉCEPTEUR ET TRADUCTEUR. L'ensemble émission-réception est constitué d'un émetteur-récepteur qui délivre une impulsion électrique très brève et de grande amplitude; la fonction réception se compose d'un amplificateur $24 \mathrm{~dB}$ variable par bonds de $1 \mathrm{~dB}$ et d'une sortie analogique. Le système fonctionne soit en réflexion, soit en transmission.

Les traducteurs utilisés sont de deux catégories. Le premier type de capteurs utilisés est constitué d'un mono-cristal de niobate de lithium taillé en coupe « $\mathrm{X}$ », collé sous vide et sous forte pression à l'extrémité d'une ligne à retard elle-même constituée d'un cylindre de silice (Puropsil A); les faces des cylindres sont parallèles à 1 minute d'angle près et polies avec une planéité de 1 frange. La métallisation des traducteurs est assurée par évaporations successives d'un alliage $\mathrm{NiCr}$ et d'or sous vide secondaire. Cette technique de fabrication procure des capteurs qui ont un bon rendement mais une bande passante étroite. Par contre ils peuvent être utilisés sur des fréquences correspondant à des harmoniques impairs. Le second type de capteurs est constitué de traducteurs céramiques et sont mécaniquement amortis de façon à couvrir une large bande passante. Quatre paires de capteurs de fréquences fondamentales de 6, 20,65 et $100 \mathrm{MHz}$ sont utilisés et couvrent une gamme de fréquence de 2 à $150 \mathrm{MHz}$ dans le cas d'un système à faible absorption. Dans le cas d'une impulsion suffisamment brève et d'un traducteur de fréquence nominale de $300 \mathrm{MHz}(300 \mathrm{MHz}$ de bande passante) le système autorise des mesures jusqu'à $400 \mathrm{MHz}$.

\subsection{VisuAlisation ET ACQUISITION DU SIGNAL. -} La visualisation des échos ultrasonores s'effectue sur l'écran d'un oscilloscope numérique à mémoire à base de temps retardée ; cette dernière fonction est indispensable car elle permet de dilater sur l'écran l'écho choisi pour acquisition. La bande passante est de $400 \mathrm{MHz}$ et le temps d'acquisition du signal (en 1024 points) varie entre quelques secondes et quelques dizaines de secondes selon la base de temps.

La numérisation du signal s'effectue avec une fréquence d'échantillonnage définie par la base de temps retardée qui détermine la fenêtre d'acquisition $T$ avec :

$T=10 a=N . \Delta t$ où $a$ est le calibre de la base de temps, $N$ le nombre d'échantillons et $\Delta t$ le temps d'échantillonnage.

La fréquence d'échantillonnage est alors: $f=1 / N \cdot \Delta t$.

D'autre part l'acquisition peut être moyennée sur 10,100 ou 1000 coups de façons à augmenter le rapport signal sur bruit. Enfin le signal numérisé peut être dilaté en temps et en amplitude de façon à centrer le maximum de points d'échantillonnage par extrapolation sur la partie utile du signal.

3.3 Système DE TRAITEMENT. - Le traitement des signaux numérisés est effectué sur un micro-ordinateur muni d'une interface IEEE permettant le transfert des données par un Bus GPIB.

\section{Résultats expérimentaux.}

Les performances du système ont été testées sur des systèmes connus : liquides purs ne présentant pas de phénomènes de relaxation, liquides purs et mélanges présentant d'importants processus relaxationnels dans une gamme importante de fréquence. 
4.1 LIQUidES PURS. - Les mesures de vitesse de propagation effectuées dans l'eau à $20^{\circ} \mathrm{C}$ ont montré un bon accord avec celles effectuées au moyen de systèmes conventionnels (Fig. 2).

Le processus de relaxation mesuré dans le 1.1.2 trichloroéthane [3] est mis en évidence comme le montre les figures 3 et 4 . Les valeurs expérimentales déterminées sont compatibles à moins de $2 \%$ pour l'absorption. Les mesures ont été effectuées au moyen de trois paires de capteurs de fréquences fondamentales 6,20 et $100 \mathrm{MHz}$.

4.2 Mélanges AQueuX D'Alcools. - Les mélanges aqueux de la série des premiers alcools primaires ont fait l'objet d'études nombreuses au laboratoire $[10,11]$ et les processus de relaxation montrent des amplitudes très importantes pour certaines concentrations d'alcools.

Ces valeurs peuvent servir de références pour tester la fiabilité en valeur absolue du système. C'est ainsi que les mesures effectuées dans un certain nombre de mélanges aqueux de n-propanol sont compatibles avec les valeurs déterminées précédemment avec des précisions également de l'ordre de $2 \%$ pour l'absorption et $0,1 \%$ pour la vitesse, c'està-dire avec une erreur relative de l'ordre de grandeur de la précision expérimentale du système.

A titre d'exemple, les figures 5 et 6 rendent compte des mesures effectuées dans le mélange n- propanol/eau à $30 \%$ et $40 \%$ d'alcool et à deux températures $0{ }^{\circ} \mathrm{C}$ et $15^{\circ} \mathrm{C}$. Les résultats se confondent sur les figures avec ceux de la référence [11].

4.3 SYSTÈMES ÉVOLUTIFS. - Certains systèmes soumis à des changements de structures ou des transitions de phase sont parfois difficiles à suivre en temps réel au moyen d'une technique appropriée.

Les techniques ultrasonores présentent l'avantage de la faible interaction avec le milieu étudié et la relative facilité de mise en œuvre.

C'est ainsi que depuis quelques années un intérêt nouveau s'est porté sur cette méthode d'investigation, en particulier dans le domaine de l'étude des transitions sol-gel.

Plus particulièrement, les ultrasons peuvent assurer le suivi de systèmes gélifiants tant pour des gels physiques comme la gélatine [12] que des systèmes chimiques comme les polyuréthanes [13], les PDMS [14] ou les résines époxydes $[15,16]$.

Dans le cas des polyuréthanes l'utilisation successives de 3 paires de traducteurs de fréquences fondamentales 6,20 et $65 \mathrm{MHz}$ a permis le suivi du processus de croissance par la détermination de l'évolution de la variation d'absorption des ondes ultrasonores (Fig. 7) à différentes fréquences. Les spectres d'absorption $\alpha / f^{2}$ à différents taux d'avancement de la réaction déduits de ces valeurs sont représentés sur la figure 8 .

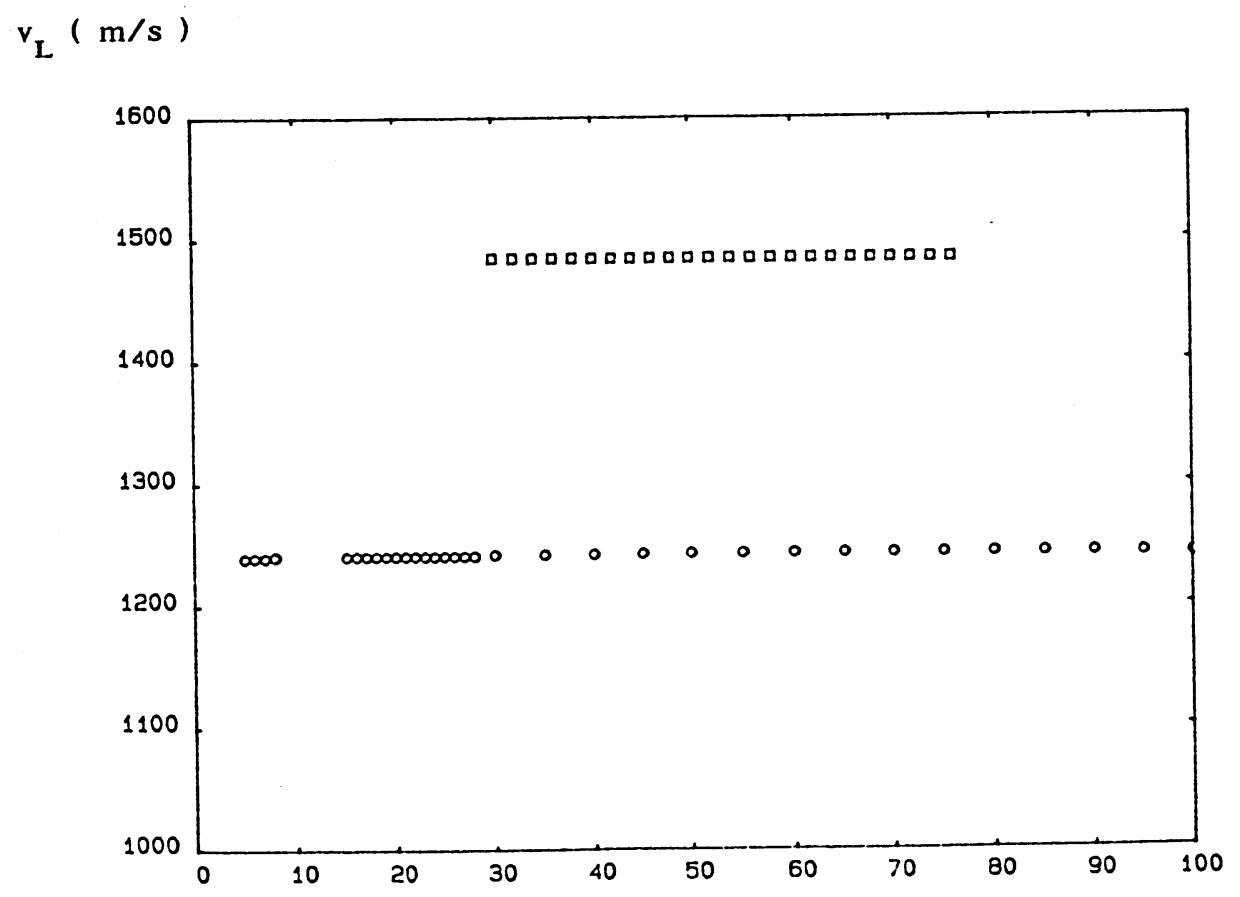

$\mathrm{f}(\mathrm{MHz})$

Fig. 2. - Vitesse de propagation des ondes longitudinales en fonction de la fréquence dans $(0)$ le $n$ propanol pur et dans (口) l'eau pure.

[Longitudinal waves velocity as a function of frequency in (O) pure n-propanol and in ( $\square$ ) pure water.] 


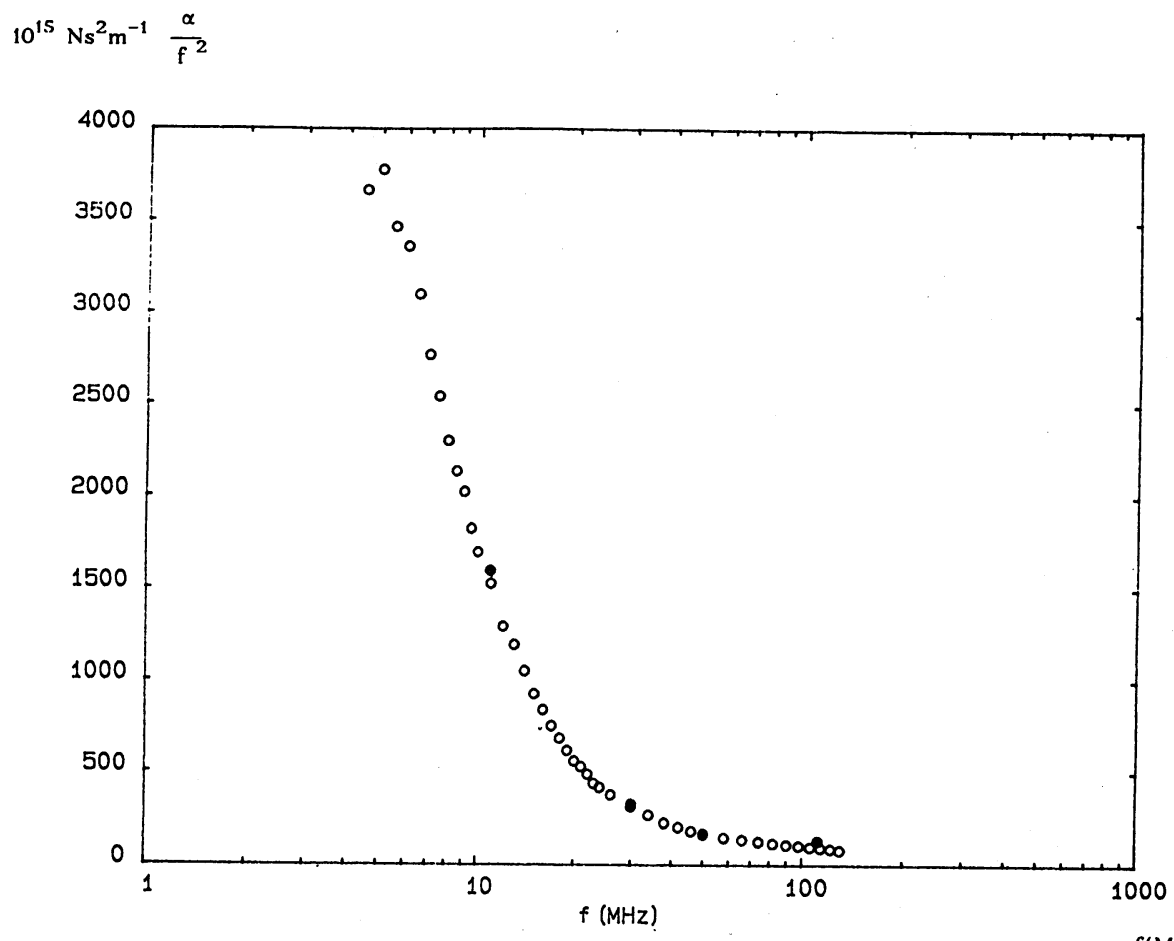

$\mathrm{f}(\mathrm{MHz})$

Fig. 3. - Spectre d'absorption dans le 1.1.2 trichloroéthane à $0^{\circ} \mathrm{C}(\bullet)$ méthode classique, $(0)$ méthode par F.F.T. [Ultrasonic absorption spectrum in 1.1 .2 trichloroethane at $0{ }^{\circ} \mathrm{C}(\bullet)$ classical method, (O) F.F.T. method.]

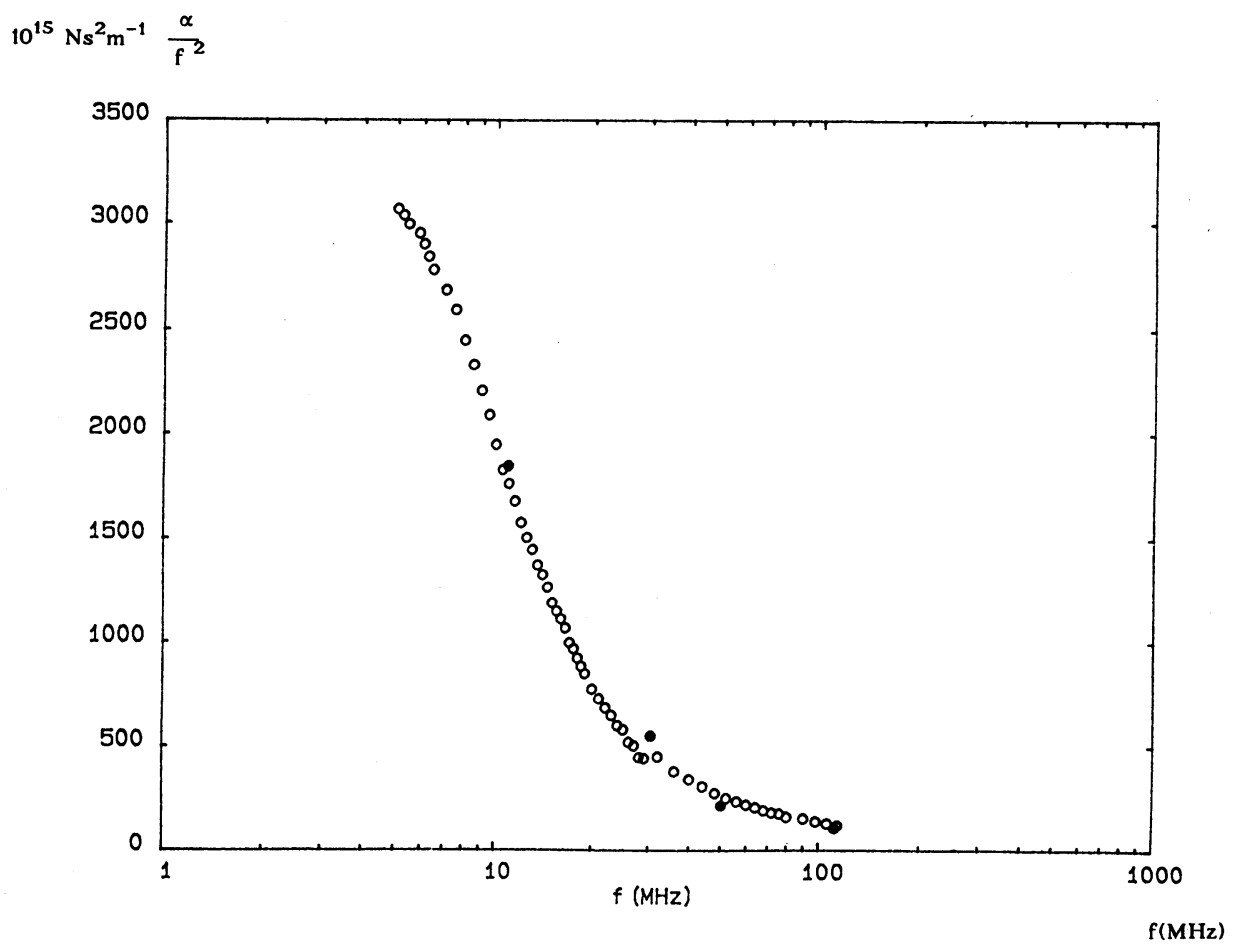

Fig. 4. - Spectre d'absorption dans le 1.1.2 trichloroéthane à $20^{\circ} \mathrm{C}(\bullet)$ méthode classique, $(O)$ méthode par F.F.T. [Ultrasonic absorption spectrum in 1.1 .2 trichloroethane at $20^{\circ} \mathrm{C}(\bullet)$ classical method, (O) F.F.T. method.] 


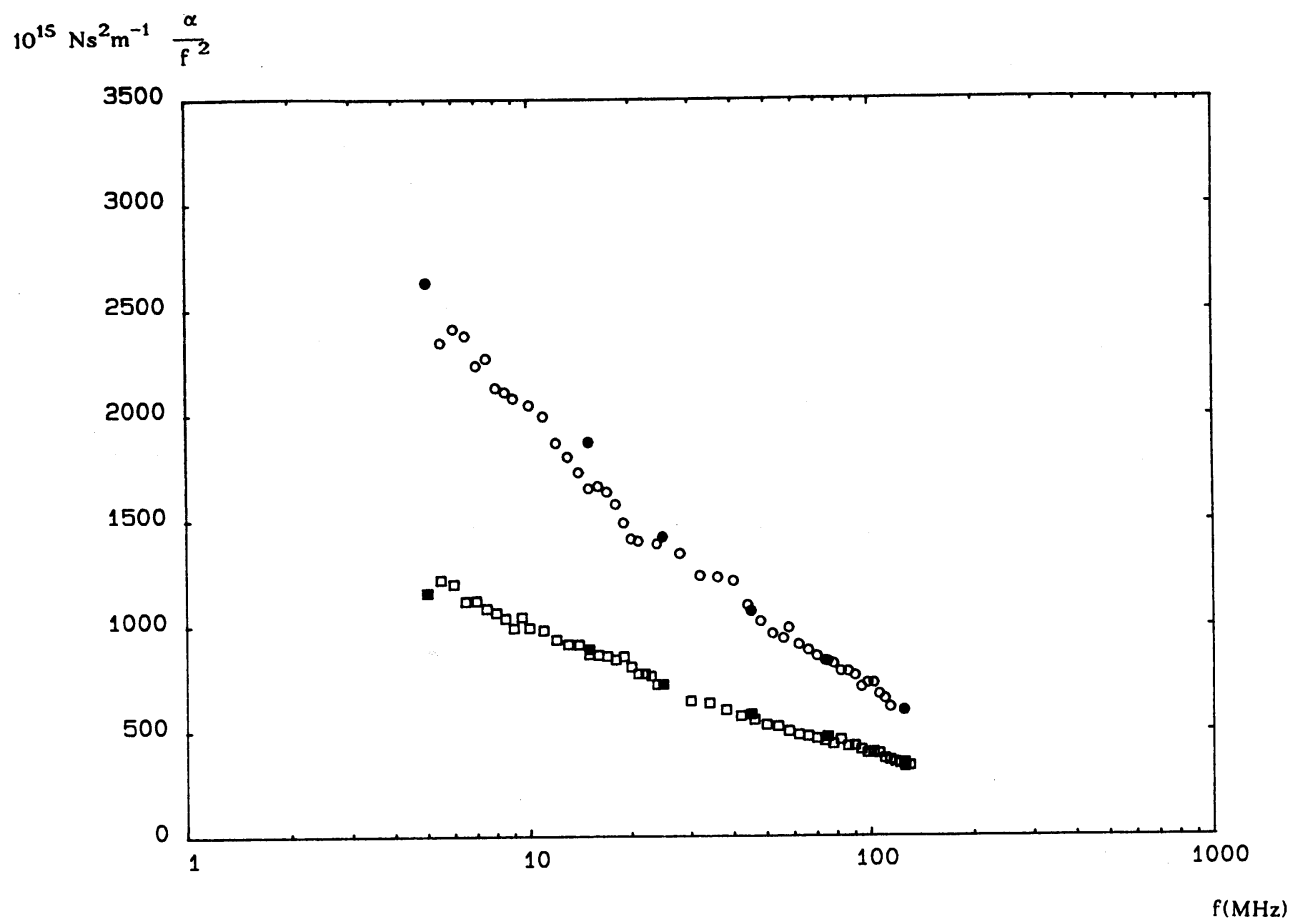

Fig. 5. - Spectres d'absorption pour les mélanges n propanol/eau à $40 \%$ d'alcool et à deux températures $(0) 0{ }^{\circ} \mathrm{C}$ et $(\square)$ $15^{\circ} \mathrm{C}$. Les symboles pleins représentent les valeurs obtenues par les méthodes classiques.

[Ultrasonic absorption spectrum in $\mathrm{n}$ propanol/water mixtures at $40 \% \mathrm{w} / \mathrm{w}$ alcohol concentration for two temperatures (O) $0{ }^{\circ} \mathrm{C}$ and $(\square) 15^{\circ} \mathrm{C}$. Filled points are data obtained by classical methods.]

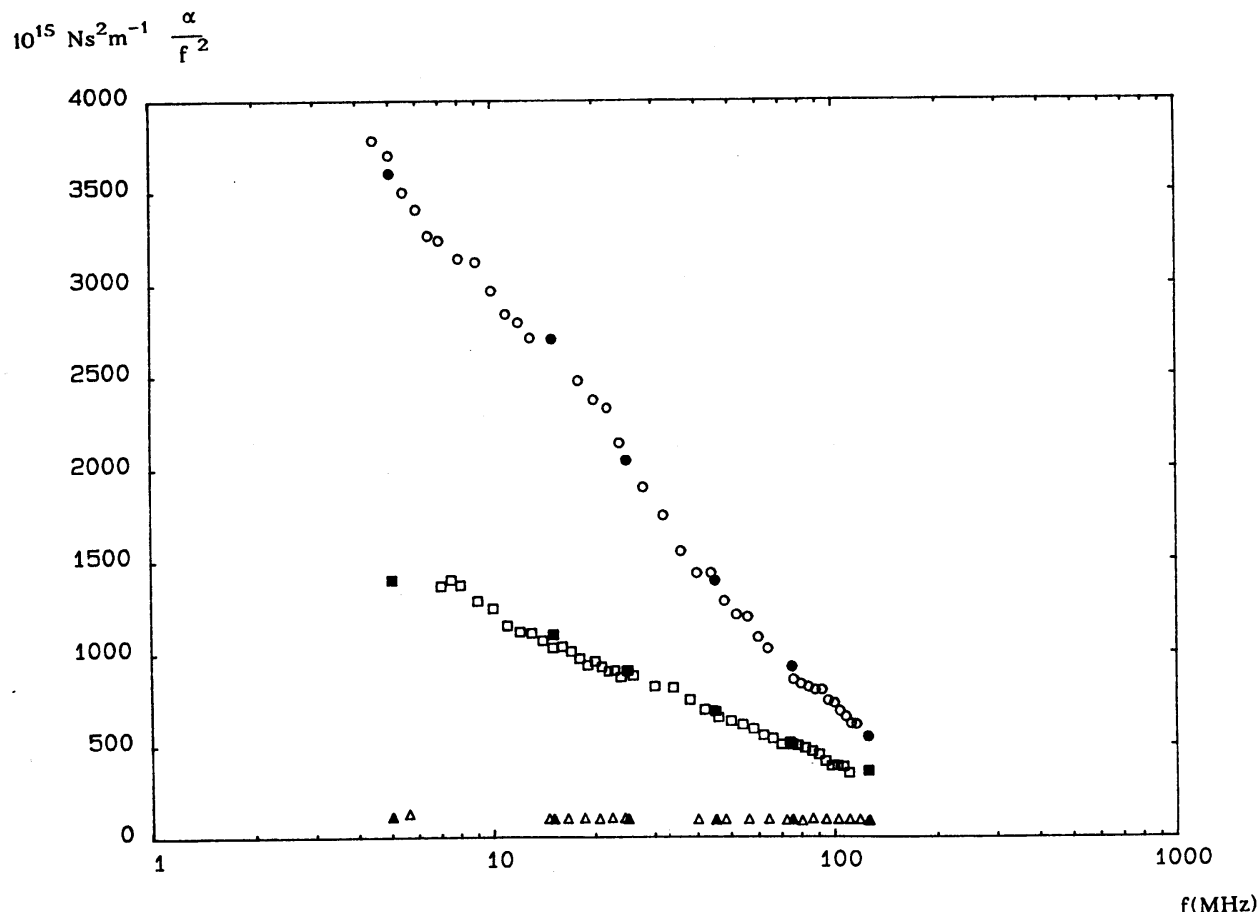

Fig. 6. - Spectres d'absorption dans le mélange n-propanol/eau à $30 \%$ d'alcool et à deux températures $(0) 0{ }^{\circ} \mathrm{C},(\square)$ $15^{\circ} \mathrm{C}$ et dans le n propanol pur à $(\triangle) 15^{\circ} \mathrm{C}$. Les symboles pleins représentent les valeurs obtenues par les méthodes classiques.

[Ultrasonic absorption spectrum in $\mathrm{n}$ propanol/water mixtures at $30 \% \mathrm{w} / \mathrm{w}$ alcohol concentration for two temperatures (O) $0{ }^{\circ} \mathrm{C}$ and $(\square) 15^{\circ} \mathrm{C}$ and in pure n propanol at $(\Delta) 15^{\circ} \mathrm{C}$. Filled points are data obtained by classical methods.] 


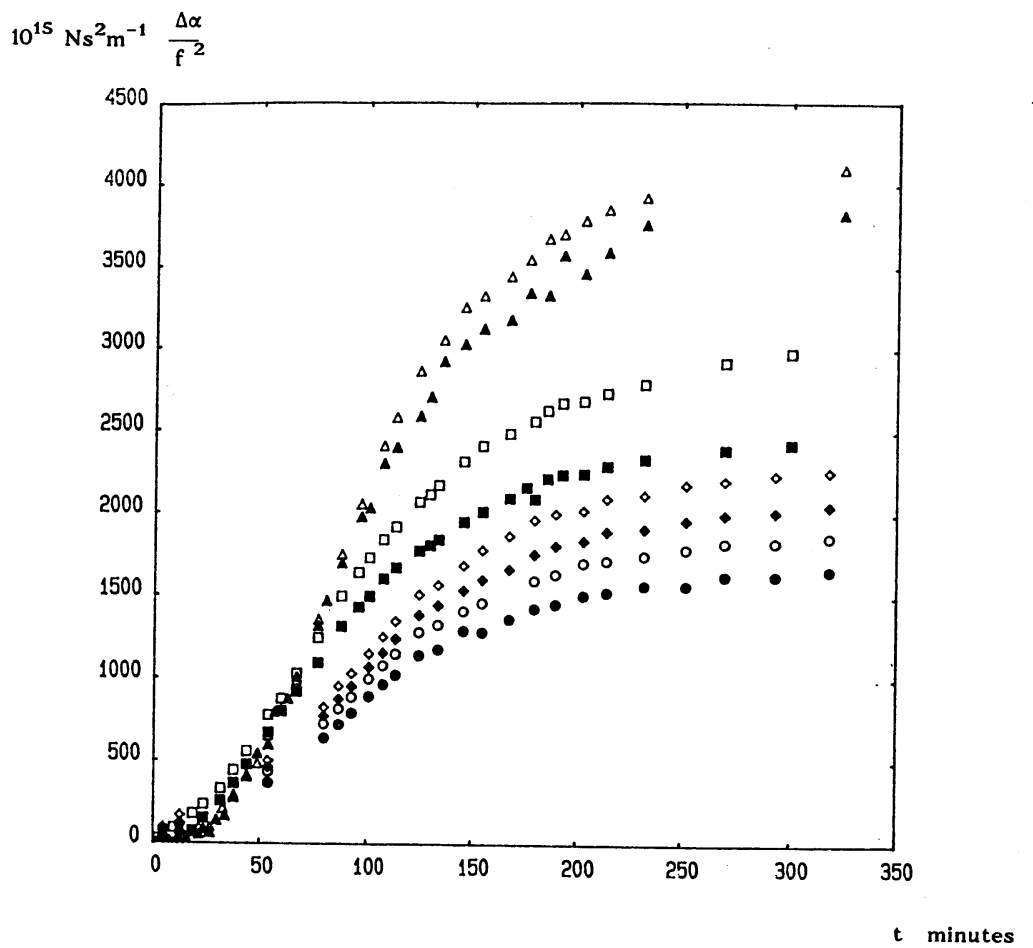

Fig. 7. - Variation d'aborption au cours de la gélification du système stœchiométrique triol700-diisocyanate (polyuréthane) dans $25 \%$ de toluène à différentes fréquences : $(\triangle) 6 \mathrm{MHz},(\Delta) 15 \mathrm{MHz},(\square) 20 \mathrm{MHz},(\square) 25 \mathrm{MHz},(\diamond)$

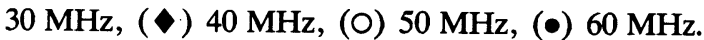

[Variation of the absorption as a function of time during the gel formation for the stoechiometric system triol 700 diisocyanate (polyurethane). Studies performed on a $25 \%$ toluene solution at different frequencies : $(\triangle) 6 \mathrm{MHz},(\Delta)$ $15 \mathrm{MHz},(\square) 20 \mathrm{MHz},(\square) 25 \mathrm{MHz},(\diamond) 30 \mathrm{MHz},(\diamond) 40 \mathrm{MHz},(\bigcirc) 50 \mathrm{MHz}$ and (•) $60 \mathrm{MHz}$.]

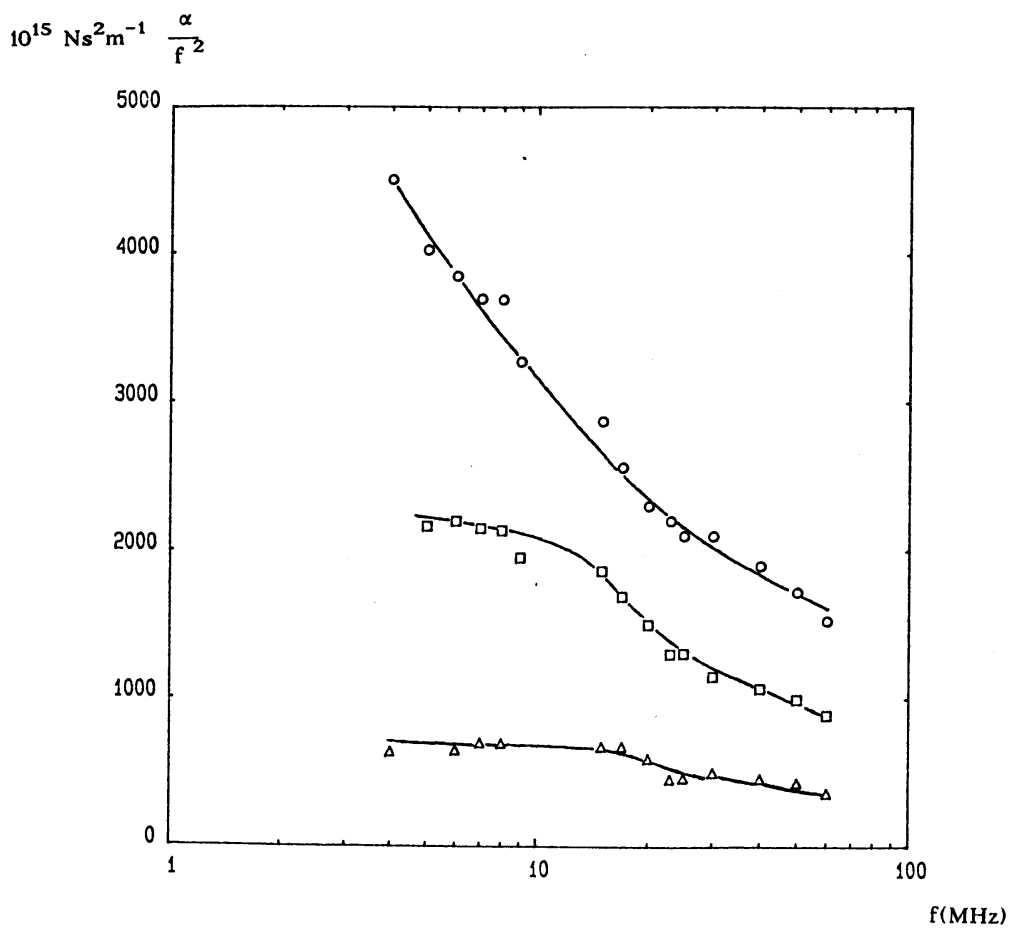

Fig. 8. - Spectres d'absorption à différents temps au cours de la gélification du système stœchiométrique triol700diisocyanate (polyuréthane) dans $25 \%$ de toluène : $(\triangle) 54 \mathrm{~min},(\square) 101 \mathrm{~min}$ et $(O) 214 \mathrm{~min}$.

[Ultrasonic absorption spectrum at different times of the gel formation for the stoechiometric system triol 700 diisocyanate (polyurethane) in $25 \%$ toluene concentration : $(\triangle) 54 \mathrm{~min}$, ( $\square$ ) $101 \mathrm{~min}$ and (O) $214 \mathrm{~min}$. 


\section{Conclusion.}

Une spectroscopie ultrasonore par FFT a été développée pour permettre d'appréhender les paramètres ultrasonores absorption et vitesse sur une large gamme de fréquences $(1-400 \mathrm{MHz})$. La validation expérimentale de cette technique a été testée sur des liquides purs et des mélanges aqueux d'alcool. Le bon accord des résultats avec ceux obtenus par les méthodes classiques autorise la détermination des spectres ultrasonores par cette méthode.

Une des finalités de la mise au point de cette technique est la détermination de l'évolution temporelle des spectres de relaxation pour des systèmes évolutifs et plus particulièrement pour des systèmes dont la croissance est gouvernée par des processus d'agrégation. C'est ainsi que l'étude des réseaux polyuréthanes a été abordée et fera l'objet d'une prochaine publication.

L'aptitude de cette technique à appréhender la totalité du processus de croissance de systèmes évolutifs conduit à penser qu'elle fournit une approche originale et un outil privilégié pour l'étude des réseaux.

\section{Remerciements.}

Les auteurs tiennent à remercier G. Ripault pour son assistance technique au cours de cette étude qui a pu être réalisée grâce à un financement du MRT . (référence 85 G113 1985-1988) et de l'INRA (ATP $\mathrm{N}^{\circ} 4412$ 1985-1988).

\section{Bibliographie}

[1] Herzfeld K. F. and Litovitz T. A., Absorption and dispersion of ultrasonic waves (Academic Press, N.Y.) 1959.

[2] LAMB J., Thermal relaxation in liquids, Physical Acoustics, Vol. II, Part A IV (1965).

[3] Pethrick R. A., Molecular Acoustics, Molecular Interactions, Vol. 3, Eds. H. Ratajckzak and J. W. Orville-Thomas (1982).

[4] Emery J. R., Gasse S., Pethrick R. A. et Phillips D. K., Adv. Mol. Rel. Inter. Proc. 12 (1978) 47.

[5] Launay J. M., Bulou A. and Nouet J., Rev. Acoust. 2 (1983) 61.

[6] Dieulesaint E., Royer D., Ondes élastiques dans les solides; Application au traitement du signal (Masson) (1974) Chap. 1.

[7] BRACEWELl R., The Fourier transform and its applications (New York, Mc Graw Hill) 1965.

[8] Kline R. A., J. Acoust. Soc. Am. 76 (1984) 498-504.
[9] Fitting D. W. and AdLeR L., Ultrasonic Spectral Analysis For Nondestructive Evaluation (Plenum Press) 1981.

[10] Emery J. R. et Gasse S., Acustica 133 (1979) 205.

[11] Gasse S. et Emery J. R., J. Chim. Phys. 77 (1980) 263-266.

[12] Emery J. R., Chatelier J. Y. et Durand D., J. Phys. France 47 (1986) 921-926.

[13] Emery J. R., Durand D., TABellout M. and Pethrick R. A., Polymer 28 (1987) 1435-1439.

[14] Sheffer A., EMERy J. R., DuRAND D., GorodetSKY G. and GotTliEB M., 9th Polymer Networks, Freiburg, 26-30 September 1988.

[15] LAIREZ D., $16^{\text {cs }}$ Journées d'études sur les polymères, oct. 88 (Chantilly).

[16] Winfree W. P., Ultrasonics Symposium (1983) pp. $866-869$. 